\title{
Efficacy of Local Methylprednisolone Injection for Treatment of de Quervain's Tenosynovitis
}

ISSN: 2576-8875

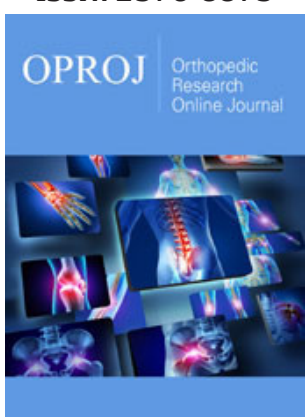

*Corresponding author: Zeeshan Khan, Department of Orthopaedics Khyber Girls Medical College, Hayatabad Medical Complex Peshawar, Pakistan

Submission: 眥 August 09, 2021

Published: 価August 23, 2021

Volume 8 - Issue 4

How to cite this article: Israr Ahmad, Sanaullah, Salik Kashif, et al. Efficacy of Local Methylprednisolone Injection for Treatment of de Quervain's Tenosynovitis. Ortho Res Online J. 8(4). OPROJ. 000693. 2021.

DOI: $10.31031 /$ OPROJ.2021.08.000693

Copyright@: Zeeshan Khan, This article is distributed under the terms of the Creative Commons Attribution 4.0 International License, which permits unrestricted use and redistribution provided that the original author and source are credited. Israr Ahmad ${ }^{1}$, Sanaullah², Salik Kashif², Zeeshan Khan²*, Mohammad Saeed
and Muhammad Arif Khan

${ }^{1}$ Associate Professor, Khyber Girls Medical College, Hayatabad Medical Complex Peshawar, Pakistan

${ }^{2}$ Department of Orthopaedics Khyber Girls Medical College, Hayatabad Medical Complex Peshawar, Pakistan

\begin{abstract}
Objectives: The objective of this study was to find out the efficacy of local steroid injection for treatment of de Quervain's disease.

Study design: Descriptive retrospective

Place and duration of study: The study was conducted at department of orthopedic and spine surgery, Hayatabad medical complex Peshawar and Khyber medical centre dabgari garden Peshawar from January 2007 to December 2019.

Patients and methods: We evaluated our data of patients with diagnosis of de Quervain's tenosynovitis from 2007 till 2019. Patients with complete demographic data and who received local steroid injection and had at least 3 months follow up were included in the study. The findings were analyzed for the effectiveness of local steroid injection. The response to injection was considered effective when the patient was symptom free and did not need surgery. Local injection of $80 \mathrm{mg}$ methylprednisole was injected into the first dorsal compartment of the wrists. The injection was repeated if first dose was not effective at $4^{\text {th }}$ or $8^{\text {th }}$ week with patients consent. The outcome was checked for relief of symptoms or otherwise at 4,8 and $12^{\text {th }}$ weeks.

Results: Complete record of 270 patients was available. Females were 238 and 32 were males in a ratio of 1:7.4.The average age was 39 years ( range 24-71). Out of these 26 patients follow up was not complete. 180 patients needed one, 49 two and 15 patients three injections. Out of 244 patients $70(28.6 \%)$ patients did not improve and needed surgical intervention. The remaining 174 ( $71.4 \%$ ) had complete relief of pain. 4 patients had hypo pigmentation of the wrist area. 28 patients had exaggerated pain for 2-3 days before the injection was effective.
\end{abstract}

Conclusion: local steroid injection is safe and effective method for treatment of de Quervain tenosynovitis. Keywords: de Quervain's disease; Tenosynovitis; Local steroid injection; [ ${ }^{\text {st }}$ dorsal compartment of wrist

\section{Introduction}

De Quervain tenosynovitis is a condition which involves tendon entrapment affecting the first dorsal compartment of the wrist [1-3]. There is thickening of the tendon sheaths of $I^{\text {st }}$ dorsal compartment of wrist located along the radial styloid. There is characteristic pain which is exacerbated by thumb movement and radial and ulnar deviation of the wrist $[2,4,5]$.

The exact cause of de Quervain tenosynovitis is not clear. This has been attributed to myxoid degeneration with fibrous tissue deposits and increased inflammation of the synovial lining. There is thickening of the tendon sheath, resulting in painful entrapment of the abductor pollicis longus and extensor pollicis brevis tendons [6,7]. It is associated with repetitive wrist motion, specifically motion requiring thumb radial abduction and extension and radial deviation. This is the basis of the Finkelstein's test. The classic patients are female manual workers or mothers of young infants $[5,8,9]$. 
The prevalence of de Quervain tenosynovitis ranges $0.5 \%$ in males and $1.3 \%$ in females with peak prevalence in their forties and fifties. Recently use of smart phones with repeated use of thumb for texting has been reported to be associated with this disease [10-12]. Common association is with other work-related musculoskeletal disorders of upper limb like, medial or lateral epicondylitis [13]. Bilateral involvement is often reported in new mothers or childcare providers [9].

The first dorsal compartment of the wrist contains the abductor pollicis longus and extensor pollicis brevis tendons. These tendons pass through a fibrous tunnel passing over the radial styloid and under the extensor retinaculum. The tendons are at risk for entrapment at the narrow space when subjected to acute trauma or repetitive motion. There is association of local anomalies with disease like duplication of tendons and tunnels [14-16].

Patients present with wrist pain, which is worsened by thumb and wrist motion. Tenderness over the radial styloid is usually present, and fusiform swelling in this region may also be noticed. The pathognomonic provocative Finkelstein test, in which the thumb is flexed and held inside a fist, and patient actively deviates the wrist to ulnar side, causes sharp pain along the radial wrist at the first dorsal compartment $[4,8,17]$. The diagnosis of de Quervain tenosynovitis is aclinical $[6,13]$. Radiographs are usually done to exclude other less common pathologies [3].

Most mild cases of de Quervain tendinopathy may resolve spontaneously. Others may need splinting, systemic antiinflammatory and corticosteroid injections [1,5,18,19]. Corticosteroid injection has been reported to provide excellent results by various researchers. Injection is performed into the tendon sheath about $1 \mathrm{~cm}$ proximal to the radial styloid where the tendons are palpable. Symptomatic relief is achieved in up to $50 \%$ of patients with a single injection $[5,6,18,19]$. A second injection may increase the response to $80 \%$ of patients. Complications of steroid injection include fat and dermal atrophy and hypo pigmentation $[4,19]$.

Surgical release of first dorsal compartment is needed if conservative treatment fails [17,20-22]. Surgery is usually done as day case, under local anaesthesia $[14,15]$. The purpose of this study is to know about the effectiveness of local methylprednisolone for treatment of de Querrvain tenosynovitis who did not respond to conservative treatment with NSAIDs and rest.

\section{Patients and Methods}

The study period was from January 2007 to December 2019. All patients were seen in outpatient in two settings, Khyber Medical Centre Dabgari Garden Peshawar and Hayatabad Medical Complex Peshawar. Data of all patients with de Quervain tenosynovitis was evaluated. Those who received local methylprednisolone and completed the required follow of 12 weeks were evaluated. The response to injection was considered effective when the patient symptoms improved and did not require surgical intervention and treatment was discontinued.

\section{Results}

Complete record of 270 patients was available. Females were 238 and 32 were males in a ratio of 1:7.4. The average age was 39 years (range 24-71) . Out of these 26 patients were lost to follow up. Most patients responded to single dose of methyl prednisolone $180(74 \%) .49$ (20\%) two and 15 (6\%) patients three injections. Out 244 patients $70(28.6 \%)$ patients did not improve despite one or two injections and needed surgical intervention. We had 174 ( $71.4 \%$ ) who improved with one two or three injections and did not require further treatment. 4 patients had hypo pigmentation of the wrist area. 28 patients had exaggerated pain for 2-3 days before the injection was effective. The response to injection was good in male patients (Figure 1-4).

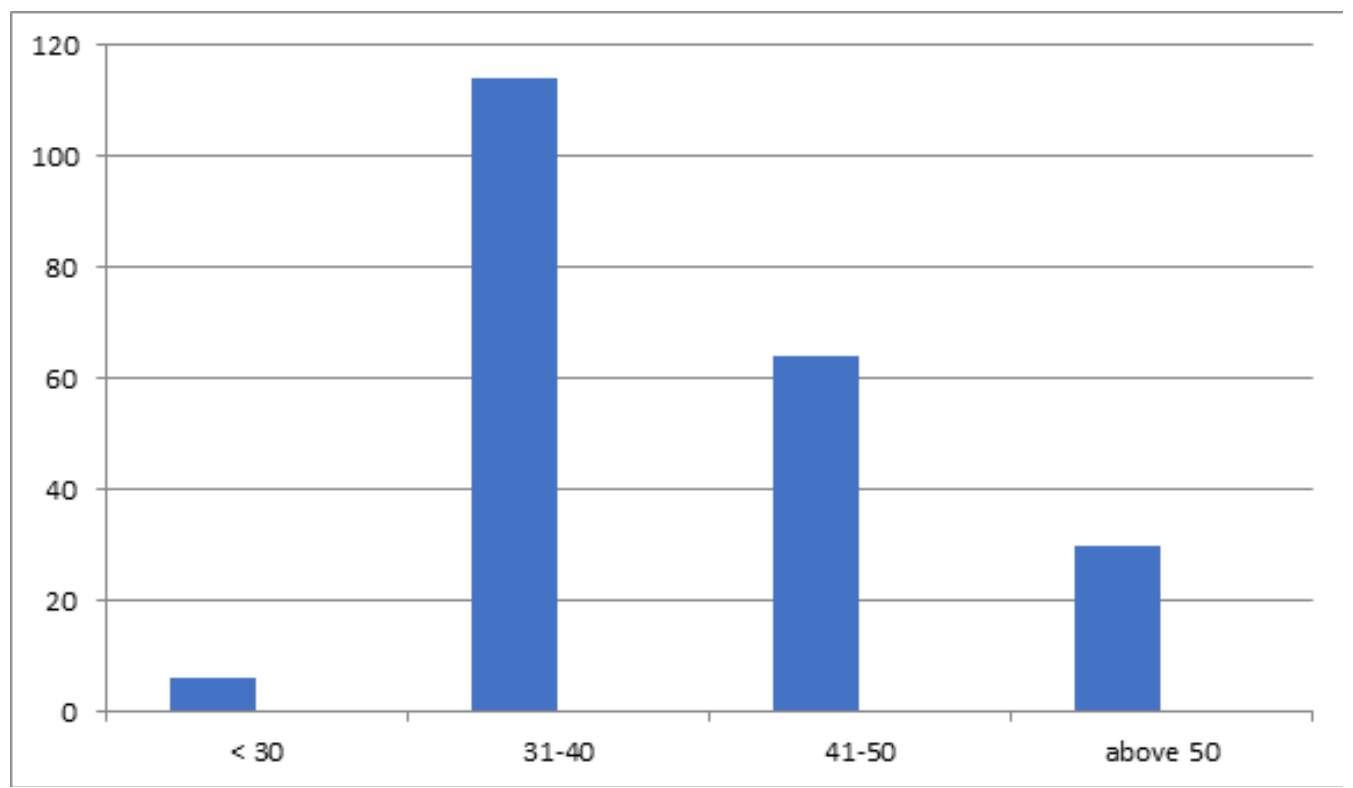

Figure 1: Age distribution of patients. 


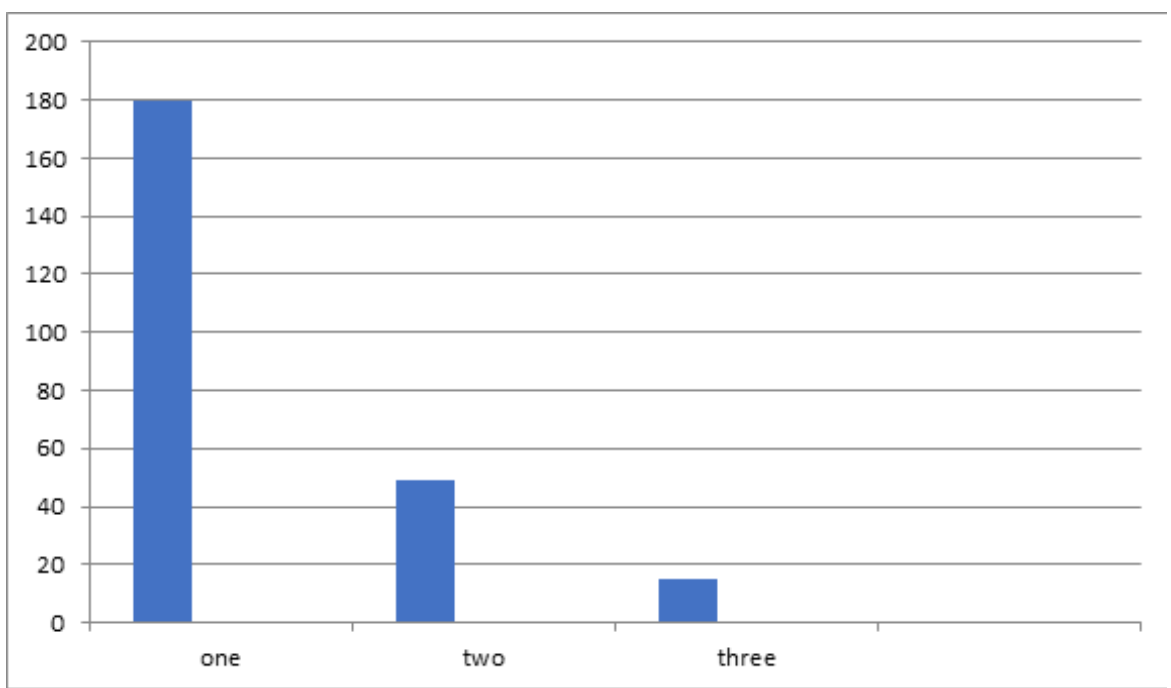

Figure 2: Frequency of injections for patients.

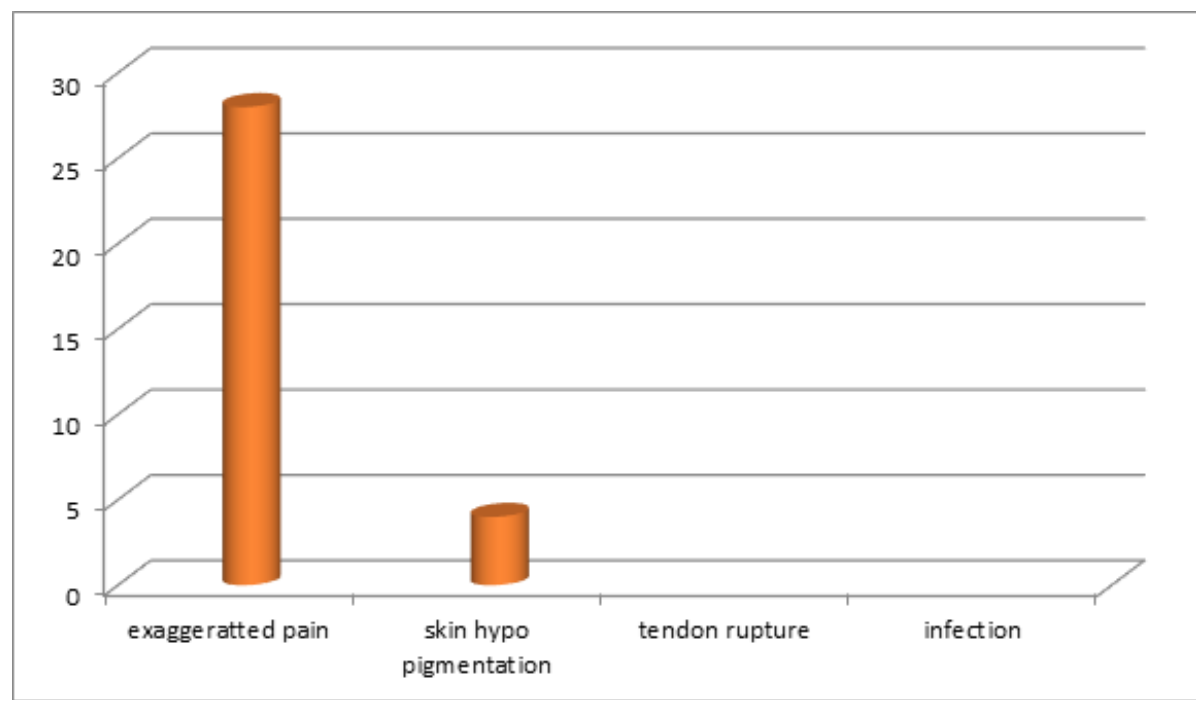

Figure 3: Side effects/ complications of injection.

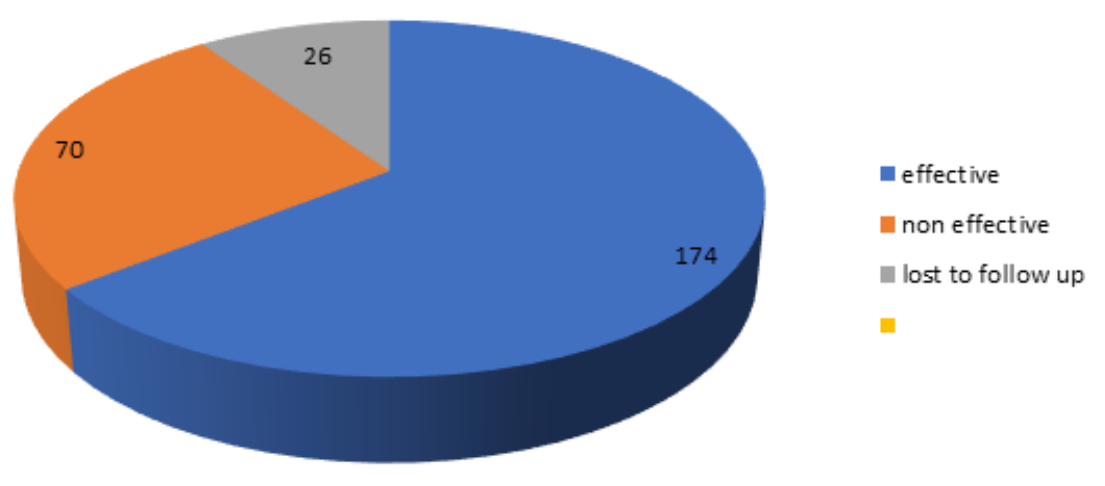

Figure 4: Efficacy of local steroid. 


\section{Discussion}

De Quervain tenosynovitis is a common work-related musculoskeletal disorder of upper limb. as There are numerous studies on various aspects from its incidence to risk factors and results of various treatment options from analgesics to surgical release. The most consistent risk factor reported by all authors is female sex.

In our study female to male ratio was 7.4:1 which is slightly lower than previous studies $[14,20]$. In our own study published in 2003 the ratio was 9:1 [17]. Some recent studies are showing change of the ratio due to increased use of smart phones as shown in a study by Samuel et al. [11]. In a recent study in Karachi by Nasim et al. [12] the ratio was almost 2:1 but they included mild cases also [12]. The female male ration is further down in this conservative group as compared to our own study in the surgical group which was 8.1. We can say that female is more prone to poor response to steroid injections [14]. The average age of our patients was 39 which is same as our previous study. This is comparable with most other studies $[1,5,17]$. Satoshi et al. [6] in their study showed that the disease was more common in forties and fifties which is similar to our study $[6,14]$.

The response to steroid injection was very effective as reported by most researchers. This has been the most effective way of treating those patients who do not respond to activity modification and NSAIDs. Our study showed above $70 \%$ efficacy which is almost same as other studies show [1,4]. In a study by Akram et al. [23] the effectiveness was above $90 \%$ after three injections, but in our case many patients opted for surgery even after one or two injections.

Steroid injection is safe as it does not result in serious systemic side effects of steroids. The most common problem we encountered was exaggerated pain response for few days which is probably due to local inflammatory response to steroid $[5,14,17]$. But it resolved within few days, and we routinely explained this to the patient later on. The other side effect was local hypo pigmentation which was encountered in 4 patients. No incidence of injection into the tendon and tendon ruptured was noted in our series [24-29].

\section{References}

1. Mehdinasab SA, Alemohammad SA (2010) Methylprednisolone acetate injection plus casting vs casting alone for treatment of de Quervain tenosynovitis. Arch Iran Med 13(4): 270-274.

2. Jaworski CA, Krause M, Brown J (2010) Rehabilitation of the wrist and hand following sports injury. Clinic Sports Med 29(1): 61-80.

3. (2017) Tenosynovitis. Skeletal Radiol 46(8): 1047-1056.

4. Rowland P, Phelon N, Galvin R (2015) The effectiveness of corticosteroid injection for De Quervain tenosynovitis(DQST): A systematic review and Metaanalysis. The Open Orthopaedic Journal 30(9): 437-444.

5. Ilyas AM (2009) Nonsurgical treatment for de Quervain's tenosynovitis. J Hand Surg Am 34(5): 928-929.

6. Satoshi A, Atsushi Y, Tsutomu K, Tsuyoshi T, Tetsuya K, et al. (2011) Prevalence of de Quervain's disease in the general population and risk factors. Kitakanto Med J 61: 479-482.
7. Clarke MT, Lyall HA, Grant JW, Matthewson MH (1998) The histopathology of de Quervain's disease. J Hand Surg Br 23(6): 732-734.

8. Moore JS (1997) De Quervain>s tenosynovitis: stenosing tenosynovitis of the first dorsal compartment. J Occup Environ Med 39(10): 990-1002.

9. Capasso G, Testa V, Maff ulli N, Turco G, Piluso G (2002) Surgical release of de Quervain's stenosing tenosynovitis postpartum: can it wait? Int Orthop 26(1): 23-25.

10. Dharti H, Dharti P, Himani D, Vidhi D (2018) Prevalence of de Quervain's tenosynovitis and its effect on pinch strength in mobile users. International Journal of Recent Scientific Research 9(3): 25032-25035.

11. Samuel DJM, Braham SS, Vincent GGA, Joshua S, David JG (2020) A review of De Quervain's stenosing tenosynovitis in the context of Smartphone use. The J hand Surg 25(2): 133-136.

12. Naseem A, Hafiz YI, Rabiya J, Tahniat W, Samra S (2019) Occurrence of De Quervain's tenosynovitis and its association with Short Message Service Texting Habit: A cross-sectional Study in the General Population of Karachi, Pakistan. International Archives of BioMedical and Clinical Research 5(1): 7-11.

13. Palmer K, Walker-Bone K, Linaker C (2009) The Southampton examination schedule for the diagnosis of musculoskeletal disorders of the upper limb. Ann Rheum Dis 59(1): 5-11.

14. Ahmad I, Hussain K, Khan Z, Kashif S, Saeed M et al. (2021) Outcome of surgical release of 1st dorsal compartment of the wrist for De quervain's tenosynovitis. Med J Clin Trials Case Stud 5(1): 000277.

15. Ahmad I, Hussain K, Khan Z, Kashif S, Saeed M, et al. (2020) Intra operative Anatomical variations of the first extensor compartment of the wrist in patients of de Quervain's disease. J Pak Orthop Assoc 32(3): 153-157.

16. Ahmad I, Khan A, Khan Z, Kashif S, Saeed M, et al. (2020) Seasonal variations and occupational risk factors: Analysis of 460 patients of de Quervain's tenosynovitis. Pak J Surg 36(3): 251-254.

17. Zarin M, Ahmad I (2003) Surgical treatment of De quervain's disease. J Coll Physicians Surg Pak 13(3): 157-158.

18. McKenzie JM (1972) Conservative treatment of de Quervain's disease. Br Med J 4(5841): 659-660.

19. Witt J, Pess G, Gelberman RH (1991) Treatment of de Quervain tenosynovitis. A prospective study of the results of injection of steroids and immobilization in a splint. J Bone Joint Surg Am 73(2): 219-222.

20. Wolf JM, Sturdivant RX, Owens BD (2009) Incidence of de Quervain's tenosynovitis in a young, active population. J Hand Surg Am 34: 112-115.

21. Roquelaure Y, Ha C, Leclerc A (2006) Epidemiologic surveillance of upper-extremity musculoskeletal disorders in the working population. Arthritis Rheum 55(5): 765-778.

22. Scheller A, Schuh R, Honle W, Schuh A (2009) Long-term results of surgical release of de Quervain's stenosing tenosynovitis. Int Orthop 33(5): 1301-1303.

23. Akram M, Shahzad ML, Farooq FM, Irshad M, Kumar Sa R, et al. (2014) Results of injection corticosteroids in treatment of De Quervain's Tenosynovitis. JPMA 64(12): 530-533.

24. Walker-Bone K, Palmer KT, Reading I, Coggon D, Cooper C (2004) Prevalence and impact of musculoskeletal disorders of the upper limb in the general population. Arthritis Rheum 51(4): 642-651.

25. Rettig AC (2004) Athletic injuries of the wrist and hand: part II: overuse injuries of the wrist and traumatic injuries to the hand. Am J Sports Med 32(1): 262-273.

26. Chang CY, Kheterpal AB, Vincentini JRT, Huang AJ (2017) Variations of anatomy on MRI of the first extensor compartment of the wrist and 
association with DeQuervain tenosynovitis. Skeletal Radiol 46(8):10471056.

27. Ta KT, Eidelman D, Thomson JG (1999) Patient satisfaction and outcomes of surgery for de Quervain's tenosynovitis. J Hand Surg Am 24(5): 10711077.
28. Mellor SJ, Ferris BD (2000) Complications of a simple procedure: de Quervain's disease revisited. Int J Clin Pract 54(2): 76-77.

29. Saeed MA, Irshad M (2010) Seasonal variation and demographic characteristics of carpal tunnel syndrome in Pakistani population. J Coll Physician Surg Pak 20(12): 798-801.

For possible submissions Click below: 